\title{
Fatores socioeconômicos interferentes na adesão ao tratamento dietoterápico de pacientes com diabetes mellitus tipo 2
}

\author{
Socioeconomic factors interfering with adherence to diet therapy for patients with type 2 \\ diabetes mellitus
}

\section{Factores socioeconómicos que interfieren con la adherencia a la terapia dietética en pacientes con diabetes mellitus tipo 2}

Andrellice Miranda Cunha ${ }^{1 *}$, Rayssa Camille Viana Pires ${ }^{1}$, Michelle de Freitas Monteiro ${ }^{1}$, Erika Cristina dos Santos Reis ${ }^{1}$, Arnaldo Junior Ramos Fonseca ${ }^{1}$, Yasmin Bentes Pinto ${ }^{1}$, Camila de Oliveira Brito ${ }^{1}$, Victor Ângelo Alves da Cruz Santos ${ }^{1}$, Pilar Maria de Oliveira Moraes ${ }^{1}$, Victor Gabriel Reis da Silva ${ }^{2}$.

\section{RESUMO}

Objetivo: Analisar evidências científicas sobre fatores socioeconômicos associados a adesão ao tratamento de pacientes portadores de diabetes, e intervenções nutricionais que promovam a adesão ao tratamento. Métodos: Trata-se de uma pesquisa descritiva do tipo revisão integrativa. Foram selecionados artigos publicados em revistas científicas disponibilizados pelas bases de dados Biblioteca Virtual de Saúde (BVS). Foram incluídos artigos publicados entre 2011 ao ano de 2021, em língua portuguesa, inglesa e espanhola. Sendo a busca com as palavras-chaves: diabetes, dietoterapia, plano alimentar e adesão ao tratamento. Resultados: Os resultados indicam que a adesão ao tratamento dietoterápico ainda é baixa $(<50 \%)$ por pacientes diabéticos, a literatura evidencia que fatores sociodemográficos como idade, escolaridade e renda são fortes fatores interferentes à adesão. Além disso demonstrou que a dietoterapia do paciente portador de diabetes mellitus tipo 2 torna-se complexa por envolver a presença de sentimentos que influenciam diretamente no tratamento da doença, nesse sentido uma adoção de intervenção motivadora não somente para os pacientes, mas também para a família pode promover maior adesão. Considerações finais: $\mathrm{A}$ adoção da educação nutricional com abordagens menos tecnicistas, escuta qualificada e valorização da cultura são mais do que necessárias para o planejamento alimentar do paciente diabético.

Palavras-chave: Diabetes, Dietoterapia, Planejamento alimentar, Adesão ao tratamento.

\begin{abstract}
Objective: To analyze scientific evidence on socioeconomic factors associated with adherence to the treatment of patients with diabetes, and nutritional interventions that promote adherence to treatment. Methods: This is an integrative review descriptive research. Articles published in scientific journals made available by the Virtual Health Library (VHL) databases were selected. Articles published between 2011 and 2021, in Portuguese, English and Spanish, were included. Being the search with the keywords: diabetes, diet therapy, meal plan and treatment adherence. Results: The results indicate that adherence to diet therapy is still low $(<50 \%)$ by diabetic patients, the literature shows that sociodemographic factors such as age, education and income are strong factors that interfere with adherence. In addition, it demonstrated that the diet therapy of patients with type 2 diabetes mellitus becomes complex because it involves the presence of feelings that directly influence the treatment of the disease, in this sense, the adoption of a motivating intervention not only for patients, but also for the family can promote greater adherence. Final considerations: The adoption of nutritional education with less technical approaches, qualified listening and appreciation of culture are more than necessary for the diabetic patient's food planning.
\end{abstract}

Keywords: Diabetes, Diet therapy, Food planning, Adherence to treatment.

${ }^{1}$ Escola Superior da Amazônia (ESAMAZ), Belém - PA. *E-mail: andrellice.nutri@outlook.com

2 Universidade da Amazônia (UNAMA), Belém - PA. 


\section{RESUMEN}

Objetivo: Analizar la evidencia científica sobre factores socioeconómicos asociados a la adherencia al tratamiento de pacientes con diabetes e intervenciones nutricionales que favorezcan la adherencia al tratamiento. Métodos: Se trata de una investigación descriptiva de revisión integradora. Se seleccionaron artículos publicados en revistas científicas puestas a disposición por las bases de datos de la Biblioteca Virtual en Salud (BVS). Se incluyeron artículos publicados entre 2011 y 2021, en portugués, inglés y español. Siendo la búsqueda con las palabras clave: diabetes, dietoterapia, plan de alimentación y adherencia al tratamiento. Resultados: Los resultados indican que la adherencia a la dietoterapia aún es baja $(<50 \%)$ por parte de los pacientes diabéticos, la literatura muestra que factores sociodemográficos como la edad, la educación y los ingresos son factores fuertes que interfieren con la adherencia. Además, demostró que la dietoterapia de los pacientes con diabetes mellitus tipo 2 se vuelve compleja porque implica la presencia de sentimientos que influyen directamente en el tratamiento de la enfermedad, en este sentido, la adopción de una intervención motivadora no solo para los pacientes, sino también para la familia.Puede promover una mayor adherencia. Consideraciones finales: La adopción de educación nutricional con enfoques menos técnicos, escucha calificada y apreciación de la cultura son más que necesarias para la planificación alimentaria del paciente diabético.

Palabra clave: Diabetes, Terapia dietética, Planificación alimentaria, Adherencia al tratamiento.

\section{INTRODUÇÃO}

Mundialmente o número de óbitos causados por diabetes tipo 2 (DM2) gira em todo de $5 \%$ ao ano, dentre essas mortes, $80 \%$ dos casos são de pessoas que residem em países subdesenvolvidos, com as principais causas associadas ao aumento da obesidade, envelhecimento populacional e ausência de atividade física decorrente da deficiência do organismo em usar adequadamente a insulina produzida, ou quando as taxas desta são inferiores as da glicemia (SARTORELLI DS e CARDOSO MA, 2006; REBELO AB e VAZ EM, 2014; IDF, 2017; SBD, 2019).

O diabetes mellitus (DM) é uma doença crônica de etiologia múltipla, com consequente queda na secreção de insulina ou da deficiência metabólica, resultando em resistência à insulina. Essa síndrome exige suporte médico para evitar possíveis complicações a longo prazo, concomitante com a educação do paciente acerca da doença de forma contínua (REBELO AB e VAZ EM, 2014).

Segundo a OMS, até 2030 estima-se que 333 milhões de pessoas no mundo serão portadores de diabetes, sendo segundo o Ministério da Saúde, cerca de 11 milhões no Brasil (ANDRADE RC et al., 2011). O tipo 1 da doença é caracterizado pela incapacidade do sistema imunológico na produção insulínica. Levando a uma baixa quantidade de insulina liberada para o corpo, resultando em altas doses de glicose no sangue. O Tipo 2 é equivalente da deficiência do organismo em usar adequadamente a insulina produzida, ou quando as taxas de insulina são inferiores as de glicemia (SBD, 2019).

Alterações demográficas, epidemiológicas e nutricionais marcadas no século passado foram definitivas para a situação atual do perfil populacional brasileiro e mundial com grave risco de doenças crônicas. Essas condições prevalentes descrevem os problemas de saúde pública como obesidade, doenças cardiovasculares, câncer e diabetes mellitus (VERMELHO LL et al., 2001).

O perfil epidemiológico do mundo sofreu uma grande alteração nos últimos anos. Anteriormente, o quadro epidemiológico do Brasil era marcado por doenças parasitarias e infecciosas, levando muitas pessoas a óbito. Atualmente, houve uma mudança brusca nesse quadro, o Brasil trilhou de um cenário de indivíduos desnutridos e suscetíveis a doenças transmissíveis ao atual de obesidade, com altas taxas de mortes por doenças crônicas não-transmissíveis. A alteração na estrutura da dieta dos indivíduos foi caracterizada pela diminuição do consumo de legumes, fibras e frutas para o aumento de alimentos industrializados, açúcares, e carboidratos refinados. Ou seja, o aumento da ingestão de alimentos com alta densidade calórica e pobre em nutrientes (SANTOS AFL e ARAUJO JWG, 2011). 
O fenômeno da transição nutricional ocorreu por fatores sociais, demográficos e econômicos. A globalização, os avanços tecnológicos, e a busca pela praticidade colaborou para a inserção de fast-foods no habito alimentar dos brasileiros. A alteração da estrutura do trabalho também foi relevante para a mudança no ato de comer. A mulher era e ainda é vista como provedora da alimentação para a família, logo a saída da mulher para o mercado de trabalho contribuiu para a mudança nas práticas alimentares. Todavia, os novos estilos de vida pouco saudáveis presentes atualmente no brasil é o principal fator etiológico da enorme incidência de obesidade na população brasileira (POPKIN BM, 2001).

Cerca de 74\% dos óbitos ocorridos entre pessoas de 30 a 69 anos de idade no Brasil no ano de 2016 foram causados por doenças cardiovasculares, diabetes, cânceres e doenças respiratórias crônicas. Essas taxas de mortalidade poderiam ser diferentes, considerando que essas doenças são ligadas a fatores de risco envolvidos com o comportamento humano, e que poderiam ser evitadas, como alimentação irregular, fumo, consumo excessivo de álcool e sedentarismo (WHO, 2018).

A obesidade representa um problema nutricional de grande ascensão nos últimos anos, sendo uma doença crônica que reduz a qualidade de vida do indivíduo e pode ser precursora de patologias associadas, que pelas suas complicações elevam as taxas de hospitalizações nos sistemas de saúde, e aumento dos recursos financeiros destinados ao tratamento das doenças dentre elas DM tipo 2 , que abrange números avassaladores em todo o mundo (DIAS JCR e CAMPOS JADB, 2008).

A prevalência do diabetes no Brasil passou de 5,5\% observado em 2006, para 7,4\% no ano de 2019. Com a alta prevalência de casos de diabetes e hipertensão, o Governo Federal através do Ministério da Saúde, criou o Programa de Hipertensão Arterial e Diabetes como política pública para controle e tratamento. Constitui-se de um programa de cadastramento e acompanhamento de hipertensos e/ou diabéticos que tem como objetivo o controle da DM e HAS garantindo uma melhor qualidade de vida. O paciente é vinculado à Unidade Básica de Saúde (UBS) e a Estratégia Saúde da Família (ESF) e é assistido continuamente por uma equipe multiprofissional (DIAS KC, et al., 2014).

A terapêutica atual do diabetes tem como objetivo controlar a glicemia, podendo ocorrer com manejo de dieta hipocalórica, prática de atividade física e medicação. Para o controle glicêmico, há diversas opções medicamentosas disponíveis: sensibilizados da ação de insulina, anti hiperglicemiantes, secretagogos, drogas anti-obesidade e insulina. Porém, o uso dessas medicações é indicado no DM2 quando a dieta ofertada e o exercício físico não forem capazes de reduzir os níveis glicêmicos do sangue. Infelizmente, ainda não se observa essa conduta, ao passo que dieta e exercício ainda são negligenciados em prol do tratamento medicamentoso (ARAÚJJO LMB, et al., 2000).

Usualmente o paciente diabético vem de histórico alimentar irregular, com excesso de carboidratos refinados, gordura satura, trans, excesso de sódio e açúcar. A principal conduta para o tratamento da diabetes é a retirada desses alimentos densos energeticamente que promovem picos de insulina. Daí existe o grande desafio para a mudança de hábitos do paciente, porque a retirada desses alimentos que são provenientes de anos de "hábitos prazerosos" gera angústia e dificuldade na inserção de alimentos saudáveis no cotidiano para o controle glicêmico (LOTTENBERG AMP, 2008).

Adesão ao tratamento é definido pelo grau de assimilação, conscientização, e incorporação no seu dia-adia de forma voluntaria as orientações dos profissionais de saúde. Para pacientes crônicos a adesão ao tratamento é bem mais complexa pela necessidade da continuidade. A extensão do tratamento contempla 3 estágios: concordância, adesão e manutenção (ASSUNÇÃO TS e URSINE PGS, 2008). É neste contexto que este estudo visou identificar e analisar, através de evidências científicas, fatores interferentes para a adesão ao tratamento dietoterápico de pacientes portadores de diabetes mellitus tipo 2.

\section{MÉTODOS}

Tratou-se de pesquisa descritiva do tipo revisão integrativa da literatura, com a obtenção de artigos publicados em revistas cientificas eletrônicas da área da saúde. Foram selecionados para este estudo artigos publicados pelas bases de dados Biblioteca Virtual de Saúde (BVS) acessados nas plataformas Literatura 
Latino-Americana e do Caribe em Ciências da Saúde (LILACS); Medical Literature Analysis and Retrieval System Online (MEDLINE); Índice Bibliográfico Espanhol em Ciências da Saúde (IBECS) e Banco de Dados em Enfermagem (BDENF) utilizando os Descritores de Saúde (DECS): Dietoterapia. Plano Alimentar. Adesão ao tratamento. e filtros conforme os critérios de inclusão para este estabelecido.

Foram incluídos na amostra deste estudo artigos que foram disponibilizados completos, gratuitos, publicados do ano de 2011 ao ano de 2021, em língua portuguesa, inglesa ou espanhola, e que fossem pertinentes ao título estabelecido para este estudo. Foram excluídos da pesquisa, artigos que não foram disponibilizados na integra, publicados antes do ano de 2011, que se encontraram em qualquer outro idioma que não os supracitados, que estavam duplicados e que possuíam conceitos antagônicos ao título estabelecido para este estudo (Figura 1).

Foi proposta, para avaliação, a classificação de seis níveis de evidências provenientes das pesquisas. Essa classificação considera abordagem metodológica do estudo, o delineamento de pesquisa empregado e o seu rigor (Quadro 1).

Figura 1 - Fluxograma de coleta de dados.

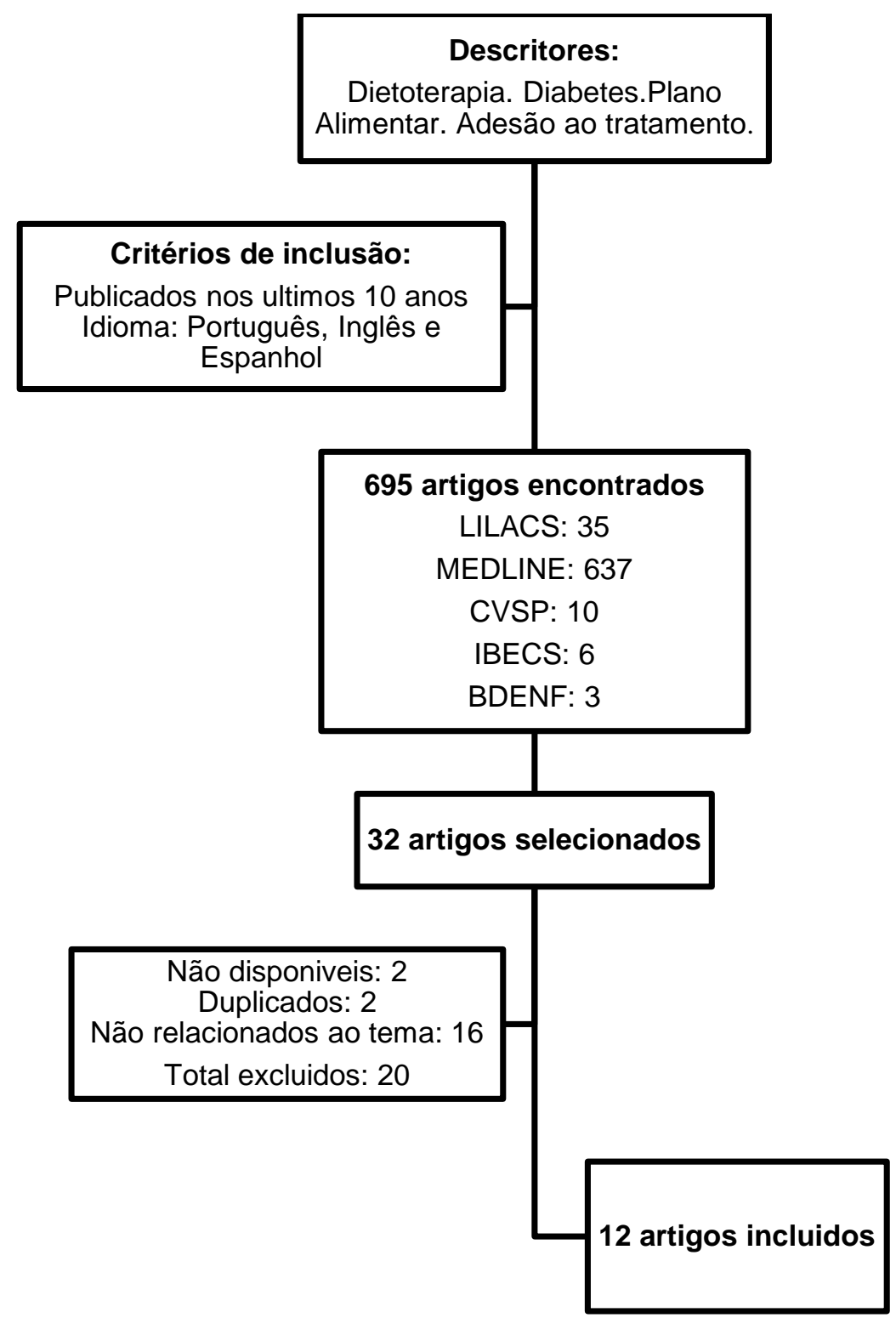

Fonte: Cunha AM, et al., 2021. 
Quadro 1 - Níveis de evidências dos artigos científicos.

\begin{tabular}{|c|c|}
\hline Nível das Evidências & Natureza do Estudo \\
\hline Nível I & $\begin{array}{c}\text { Evidências oriundas de revisão sistemática ou meta-análise de todos } \\
\text { relevantes ensaios clínicos randomizados controlados ou provenientes } \\
\text { de diretrizes clínicas baseadas em revisões sistemáticas de ensaios } \\
\text { clínicos randomizados controlados. }\end{array}$ \\
\hline Nível II & $\begin{array}{l}\text { Evidências derivadas de pelo menos um ensaio clínico randomizado } \\
\text { controlado bem delineado. }\end{array}$ \\
\hline Nível III & $\begin{array}{l}\text { Evidências obtidas de estudos quase-experimentais, como ensaio } \\
\text { clínico não randomizado, grupo único pré e pós-teste, séries temporais } \\
\text { ou caso-controle. }\end{array}$ \\
\hline Nível IV & $\begin{array}{c}\text { Evidências originárias de estudos não experimentais, como pesquisa } \\
\text { descritiva, correlacional e comparativa, pesquisas com abordagem } \\
\text { metodológica qualitativa e estudos de caso. }\end{array}$ \\
\hline Nível V & $\begin{array}{l}\text { Evidências oriundas de dados de avaliação de programas, dados } \\
\text { obtidos de forma sistemática. }\end{array}$ \\
\hline Nível VI & $\begin{array}{c}\text { Evidências a partir de opiniões de especialistas, relatos de experiência, } \\
\text { consensos, regulamentos e legislações. }\end{array}$ \\
\hline
\end{tabular}

Fonte: Cunha AM, et al., 2021; dados extraídos de Oxford Centre for Evidence-Based Medicine, 2001.

\section{RESULTADOS E DISCUSSÃO}

Foram encontrados um total de 695 artigos, sendo excluídos 492 após aplicar os critérios previamente estabelecidos na busca. $\mathrm{Na}$ base de dados LILACS, foram encontrados 16 artigos; após a avaliação dos resumos, 8 dos artigos atendeu aos critérios propostos. Na base de dados BDENF, localizou-se 2 artigos, entretanto, após análise, apenas um se adequava aos critérios de inclusão. No MEDLINE, foram encontrados 9 artigos; após a avaliação dos resumos, dois artigos foram incluídos. Nas bases de dados IBECS, foram encontrados 4 artigos, e após a avaliação, somente um foi incluído. Desta forma, totalizou-se uma amostra final de 12 artigos extraídos das bases de dados LILACS; BDENF; MEDLINE e IBECS.

A partir da análise dos textos selecionados, tem-se quanto ao tipo de publicação que os 12 estudos apresentados são nomeados pelos editores como originais. Quanto ao ano de publicação, os artigos foram publicados entres os anos de 2011 e 2020, sendo 11 em idioma português, 2 em espanhol e 1 em inglês, esses foram publicados em revistas específicas de nutrição, enfermagem e medicina.

Os objetivos descritos enquadraram-se na metodologia proposta obtendo respostas coerentes. Nesta revisão, evidenciam-se estudos quase-experimentais, observacional descritivo, observacional seccional, de corte prospectivo, estudos transversais de forma sistemática e estudos longitudinais, conforme pode ser observado abaixo (Quadro 2). 
Quadro 1 - Caracterização dos artigos selecionados para análise sobre os fatores socioeconômicos e nutricionais interferentes na adesão ao tratamento de pacientes com diabetes Mellitus tipo 2.

\begin{tabular}{|c|c|c|c|c|c|c|}
\hline Título & Autores & Idioma & Revista & Objetivo & $\begin{array}{c}\text { Nível de } \\
\text { evidência }\end{array}$ & Principais resultados \\
\hline $\begin{array}{l}\text { Adesão ao aconselhamento } \\
\text { nutricional para o diabetes } \\
\text { mellitus em serviço de atenção } \\
\text { primária à saúde. }\end{array}$ & $\begin{array}{l}\text { Rodriguez } \\
\text { MTGP, et al. } \\
(2014)\end{array}$ & Português & $\begin{array}{l}\text { REME - Revista } \\
\text { Mineira de } \\
\text { Enfermagem }\end{array}$ & $\begin{array}{l}\text { Analisar a adesão ao aconselhamento } \\
\text { nutricional em pacientes com diabetes } \\
\text { mellitus atendidos em serviço de Atenção } \\
\text { Primária à Saúde. }\end{array}$ & $\begin{array}{c}\text { Nível de } \\
\text { evidencia III }\end{array}$ & $\begin{array}{l}\text { Identificou-se que } 63,6 \% \text { dos indivíduos } \\
\text { manifestaram baixa adesão, citando a } \\
\text { restrição alimentar como a principal } \\
\text { barreira. }\end{array}$ \\
\hline $\begin{array}{l}\text { Prática alimentar e diabetes: } \\
\text { desafios para a vigilância em } \\
\text { saúde. }\end{array}$ & $\begin{array}{l}\text { Santos AFL } \\
\text { e Araújo } \\
\text { JWG (2011) }\end{array}$ & Português & $\begin{array}{l}\text { Epidemiologia e } \\
\text { Serviços de Saúde }\end{array}$ & $\begin{array}{l}\text { Caracterizar as dificuldades encontradas } \\
\text { para mudanças de práticas alimentares de } \\
\text { pessoas com diabetes mellitus tipo } 2 \\
\text { atendidas em consultório de nutrição de um } \\
\text { ambulatório de alta complexidade. }\end{array}$ & $\begin{array}{c}\text { Nível de } \\
\text { evidência IV }\end{array}$ & $\begin{array}{l}\text { O estudo detectou o uso frequente da } \\
\text { medicina popular na tentativa de cura ou } \\
\text { melhora da doença. }\end{array}$ \\
\hline $\begin{array}{l}\text { Promoção da saúde e } \\
\text { diabetes: discutindo a adesão } \\
\text { e a motivação de indivíduos } \\
\text { diabéticos participantes de } \\
\text { programas de saúde. }\end{array}$ & $\begin{array}{l}\text { Assis CJ, et } \\
\text { al. (2011) }\end{array}$ & Português & $\begin{array}{c}\text { Ciência \& Saúde } \\
\text { Coletiva }\end{array}$ & $\begin{array}{l}\text { Analisar informações publicadas sobre a } \\
\text { adesão e a motivação de pessoas } \\
\text { portadoras de diabetes mellitus } \\
\text { participantes de programas de saúde que } \\
\text { visam ao estímulo à adoção de um estilo de } \\
\text { vida saudável. }\end{array}$ & $\begin{array}{l}\text { Nível de } \\
\text { evidencia I }\end{array}$ & $\begin{array}{l}\text { Os fatores comportamentais e } \\
\text { emocionais apresentados por paciente } \\
\text { devem ser considerados no } \\
\text { planejamento de ações de saúde para } \\
\text { assistência integral a essa população. }\end{array}$ \\
\hline $\begin{array}{l}\text { Barriers to adherence to a } \\
\text { nutritional plan and strategies } \\
\text { to overcome them in patients } \\
\text { with type } 2 \text { diabetes mellitus; } \\
\text { results after two years of follow- } \\
\text { up. }\end{array}$ & $\begin{array}{l}\text { Landa-Anell } \\
\text { MV, et al. } \\
\quad(2020)\end{array}$ & Inglês & $\begin{array}{l}\text { Endocrinologia, } \\
\text { Diabetes y Nutricion }\end{array}$ & $\begin{array}{l}\text { Relatar as mudanças longitudinais na } \\
\text { percepção das barreiras à adesão ao plano } \\
\text { nutricional no DM2. }\end{array}$ & $\begin{array}{c}\text { Nível de } \\
\text { evidência IV }\end{array}$ & $\begin{array}{l}\text { A identificação de barreiras à adesão a } \\
\text { um plano nutricional pode permitir que } \\
\text { os profissionais de saúde elaborem } \\
\text { intervenções com os componentes } \\
\text { comportamentais específicos } \\
\text { necessários para superar essa barreira, } \\
\text { melhorando assim a adesão ao plano } \\
\text { nutricional com as mudanças } \\
\text { resultantes em longo prazo. }\end{array}$ \\
\hline $\begin{array}{l}\text { A adesão ao autocuidado } \\
\text { influencia parâmetros } \\
\text { bioquímicos e antropométricos } \\
\text { de pacientes diabéticos tipo } 2 \\
\text { atendidos no programa } \\
\text { Hiperdia do município de Juiz } \\
\text { de Fora, Minas Gerais, Brasil. }\end{array}$ & $\begin{array}{l}\text { Santos GFP, } \\
\text { et al. (2014) }\end{array}$ & Português & $\begin{array}{c}\text { Nutricíon Clínica y } \\
\text { Dietética Hospitalaria }\end{array}$ & $\begin{array}{l}\text { Associar a adesão ao autocuidado dos } \\
\text { pacientes diabéticos em tratamento com } \\
\text { parâmetros bioquímicos e antropométricos. }\end{array}$ & $\begin{array}{c}\text { Nível de } \\
\text { evidência V }\end{array}$ & $\begin{array}{l}\text { Pacientes que apresentaram maior } \\
\text { adesão possuíram menor valores de } \\
\text { HbA1c e apresentam menor } \\
\text { probabilidade de obterem valores de } \\
\text { HbA1c acima do valor de referência de } \\
7 \%(A U) \text {. }\end{array}$ \\
\hline $\begin{array}{l}\text { Estilo de vida en pacientes con } \\
\text { Diabetes Mellitus tipo } 2 \text { que } \\
\text { asisten al programa Regional } \\
\text { de Diabetes del Hospital } \\
\text { Regional de Encarnación } \\
\text { utilizando la encuesta IMEVID. }\end{array}$ & $\begin{array}{l}\text { Britez } \\
\text { Arévalos GN } \\
(2017)\end{array}$ & Espanhol & Tese de Mestrado & $\begin{array}{l}\text { Descrever as características demográficas } \\
\text { e clínicas, identificar a frequência do } \\
\text { consumo alimentar, e práticas relacionadas } \\
\text { à alimentação. }\end{array}$ & $\begin{array}{c}\text { Nível de } \\
\text { evidência IV }\end{array}$ & $\begin{array}{l}\text { Foi encontrada associação significativa } \\
\text { entre sexo, atividade física e emoções } \\
\text { com estilo de vida nos pacientes } \\
\text { diabéticos. }\end{array}$ \\
\hline
\end{tabular}




\begin{tabular}{|c|c|c|c|c|c|c|}
\hline Título & Autores & Idioma & Revista & Objetivo & $\begin{array}{l}\text { Nível de } \\
\text { evidência }\end{array}$ & Principais resultados \\
\hline \begin{tabular}{lrr} 
Adesão às & \multicolumn{2}{c}{ recomendações } \\
nutricionais e e variáveis \\
sociodemográficas & em \\
pacientes com & diabetes \\
mellitus. & & \\
\end{tabular} & $\begin{array}{l}\text { Zanetti ML, } \\
\text { et al. }(2015)\end{array}$ & Português & $\begin{array}{l}\text { Revista da Escola de } \\
\text { Enfermagem da USP }\end{array}$ & $\begin{array}{l}\text { Verificar se há relação entre a adesão às } \\
\text { recomendações nutricionais e variáveis } \\
\text { sociodemográficas de pacientes brasileiros } \\
\text { com DM2. }\end{array}$ & $\begin{array}{c}\text { Nível de } \\
\text { evidência IV }\end{array}$ & $\begin{array}{l}\text { A adesão às recomendações } \\
\text { nutricionais foi associada ao sexo } \\
\text { feminino, escolaridade superior a quatro } \\
\text { anos e renda familiar inferior a dois } \\
\text { salários mínimos. }\end{array}$ \\
\hline $\begin{array}{l}\text { Adesão ao tratamento dietético } \\
\text { em portadores de diabetes } \\
\text { mellitus assistidos pela } \\
\text { estratégia saúde da família. }\end{array}$ & $\begin{array}{l}\text { Santos TBM } \\
\text { e Freitas } \\
\text { BJSA (2018) }\end{array}$ & Português & Ciências da Nutrição & $\begin{array}{l}\text { Avaliar a adesão ao tratamento dietético } \\
\text { em portadores de diabetes mellitus } \\
\text { assistidos pela estratégia saúde da família, } \\
\text { verificando a existência de associação } \\
\text { entre o tipo de diabetes mellitus e as } \\
\text { práticas alimentares, condições } \\
\text { socioeconômicas e aspectos clínicos } \\
\text { relativos à doença. }\end{array}$ & $\begin{array}{l}\text { Nível de } \\
\text { evidencia V }\end{array}$ & $\begin{array}{l}\text { Concluiu-se, que os portadores de } \\
\text { diabetes mellitus recebiam orientação } \\
\text { médica e nutricional relacionada à } \\
\text { afecção, mas fatores socioeconômicos } \\
\text { e culturais, aspectos pessoais e o } \\
\text { acesso aos serviços de saúde poderiam } \\
\text { exercer influência sobre o seguimento } \\
\text { de autocuidado. }\end{array}$ \\
\hline $\begin{array}{l}\text { Educação nutricional no } \\
\text { tratamento do diabetes na } \\
\text { atenção primária à saúde: } \\
\text { vencendo barreiras. }\end{array}$ & $\begin{array}{l}\text { Moura PC, } \\
\text { et al. 2018) }\end{array}$ & Português & Revista de APS & $\begin{array}{l}\text { Avaliar um programa de educação } \\
\text { nutricional diferenciado para Estratégias de } \\
\text { Saúde da Família e identificar fatores que } \\
\text { dificultam o seguimento do plano alimentar. }\end{array}$ & $\begin{array}{l}\text { Nível de } \\
\text { evidência IV }\end{array}$ & $\begin{array}{l}\text { Não houve associação entre dificuldade } \\
\text { em seguir a dieta com as variáveis } \\
\text { renda, escolaridade e estado civil. }\end{array}$ \\
\hline $\begin{array}{l}\text { Valoración de la adherencia del } \\
\text { paciente diabético al consejo } \\
\text { nutricional y evaluación de } \\
\text { mejoras tras su reeducación: } \\
\text { una investigación-acción en la } \\
\text { costa mediterrânea. }\end{array}$ & $\begin{array}{l}\text { Lillo CM e } \\
\text { Rodríguez } \\
\text { PP (2018) }\end{array}$ & Espanhol & $\begin{array}{l}\text { Revista Chilena de } \\
\text { Nutrición }\end{array}$ & $\begin{array}{l}\text { Avaliar a adesão às recomendações } \\
\text { dietéticas entre pacientes diabéticos tipo } 2 \\
\text { e, em seguida, avaliar melhorias após } \\
\text { educação nutricional com hábitos } \\
\text { saudáveis. }\end{array}$ & $\begin{array}{l}\text { Nível de } \\
\text { evidência IV }\end{array}$ & $\begin{array}{l}\text { A não adesão alimentar pode ser } \\
\text { explicada pela ausência de um canal de } \\
\text { comunicação efetivo entre paciente- } \\
\text { família-profissional e ausência de } \\
\text { vínculo terapêutico, diminuindo o } \\
\text { empoderamento e o nível de gravidade } \\
\text { percebida pela pelo paciente e não foi } \\
\text { detectado correlação entre nível } \\
\text { acadêmico, e socioeconômico a adesão } \\
\text { à dieta. }\end{array}$ \\
\hline $\begin{array}{l}\text { Alimentação e diabetes } \\
\text { mellitus: Percepção e consumo } \\
\text { alimenta de idosos no interior } \\
\text { de Pernambuco. }\end{array}$ & $\begin{array}{l}\text { Barbosa } \\
\text { MAG, et al. } \\
(2015)\end{array}$ & Português & $\begin{array}{l}\text { Revista Brasileira em } \\
\text { Promoção da Saúde }\end{array}$ & $\begin{array}{l}\text { Avaliar o conhecimento sobre alimentação } \\
\text { relacionada ao diabetes e identificar fatores } \\
\text { que possam interferir na adesão à terapia } \\
\text { nutricional e nas escolhas alimentares. }\end{array}$ & $\begin{array}{c}\text { Nível de } \\
\text { evidencia IV }\end{array}$ & $\begin{array}{l}\text { Considerou-se adequado o nível de } \\
\text { conhecimento em } \\
\text { à alimentação e diabetes, mas viu-se } \\
\text { que fatores socioeconômicos e culturais } \\
\text { podem interferir na adesão } \\
\text { à dietoterapia. }\end{array}$ \\
\hline $\begin{array}{l}\text { Adesão ao tratamento dietético } \\
\text { e evolução nutricional e clínica } \\
\text { de pacientes com diabetes } \\
\text { mellitus tipo } 2 \text {. }\end{array}$ & $\begin{array}{l}\text { Oliveira LM, } \\
\text { et al. (2016) }\end{array}$ & Português & HU Revista & $\begin{array}{l}\text { Avaliar a adesão ao tratamento dietético e } \\
\text { a evolução do estado nutricional e clínico } \\
\text { de pacientes diabéticos assistidos pelo } \\
\text { Ambulatóriode Nutrição do Hospital } \\
\text { Universitário de Sergipe. }\end{array}$ & $\begin{array}{l}\text { Nível de } \\
\text { evidencia V }\end{array}$ & $\begin{array}{l}\text { Observou-se que apenas } 13,3 \% \text { da } \\
\text { amostra aderiram ao plano alimentar } \\
\text { proposto e que não houve evolução } \\
\text { significativa do perfil antropométrico e } \\
\text { bioquímico dos pacientes participantes } \\
\text { do estudo. }\end{array}$ \\
\hline
\end{tabular}

Fonte: Cunha AM, et al., 2021; dados extraídos de Biblioteca Virtual de Saúde (BVS). 
Em 7 dos estudos analisados foi verificado que cerca de $70 \%$ dos entrevistados em todas as pesquisas eram mulheres. A prevalência do sexo feminino na maioria dos estudos encontra respaldo na obra de Assunção TS e Ursine PGS (2008), constatando que as mulheres são mais acometidas por DM 2 do que os homens. Esses dados podem inferir que a presença feminina como maioria nas amostras revela que há maior preocupação com a saúde e consequentemente maior frequência nos serviços públicos.

A variável escolaridade em quatro artigos evidenciou uma prevalência de baixa escolaridade como fator presente na vida dos pacientes diabéticos. Zanetti ML, et tal. (2015) elucida que pacientes diabéticos com 4 anos ou mais de estudos, após serem submetidos a orientação nutricional obtiveram mais chances de fracionar as refeições do que aqueles com menor grau de escolaridade, o que sugere a necessidade de um maior grau de conhecimento para melhor compreensão e adesão ao tratamento.

Já no estudo Lillo CM e Rodríguez PP (2018), realizado na Espanha, não foi verificado relação entre escolaridade e adesão, muito embora, $70 \%$ dos entrevistados na pesquisa possuía nível superior. Nesse sentido, é necessário que ações de educação nutricional em diabetes sejam redirecionadas com maior atenção aos pacientes devendo ser adequada a linguagem considerando os diversos públicos para êxito no tratamento.

Em relação à idade, os estudos demonstram que pessoas entre 50 a 60 anos são os mais acometidos por DM 2 A idade pode ser fator interferente na aderência ao tratamento, no sentido do autocuidado, entendimento e percepção sobre a doença. Logo com o público idoso, estratégias mais simples são as mais indicadas (BARBOSA MAG, et al.,2015).

Outro fator importante é a situação econômica em que este paciente está inserido. Renda e desemprego são variáveis persistentes na baixa adesão ao tratamento. Assunção TS e Ursine PGS (2008), verificaram baixa adesão ao tratamento dietoterápico por pacientes diabéticos que residiam em locais de alto risco com territórios com alta vulnerabilidade social e pertencentes de baixa classe social, comprovando que o acesso escasso ao alimento por pessoais de mais baixa renda é fator determinantemente negativo ao tratamento do diabetes. Segundo Costa JA, et al. (2011) as limitações econômicas surgem como fator limitante para a obtenção de determinados alimentos prescritos no plano alimentar (ZANETTI ML, et al., 2015).

A Organização Mundial de Saúde (OMS, 2003), preconiza como valor maior que 50\% como boa adesão e inferior a 50\% como baixa adesão dos indivíduos na adoção dos hábitos orientados. Nesse sentido, tem se observado baixa adesão ao tratamento, principalmente no contexto à adesão a dieta prescrita para o controle glicêmico. Durco ES (2009) elucida que a baixa adesão ao tratamento é um grande impasse enfrentado pelos profissionais de saúde. A baixa aderência além de provocar frustrações pelos objetivos não alcançados, também eleva os números de hospitalizações, internações e gastos nos cuidados a saúde.

O plano alimentar para diabéticos deve ser elaborado de maneira individualizada, de acordo com as necessidades diárias, rotina de atividades físicas e hábitos alimentares. Normalmente o paciente com DM2 tende a ser obeso, então se faz necessária a adequação da dieta com o objetivo de perda de peso, e controle da doença. A prescrição de uma dieta hipocalórica é capaz de melhorar a sensibilidade à insulina e reduzir a hiperglicemia, mesmo que a perda de peso não seja observada (ARAÚJO LMB, et al., 2000). Os planos de intervenção apresentados nos estudos obtiveram em sua maioria baixa adesão $(<50 \%)$, mesmo resultado encontrado em Gomes-Villas Boas LC, et al. (2012) que constatou baixa adesão ao tratamento nutricional em 162 pacientes diabéticos estudados.

As justificativas mais relatadas por parte dos entrevistados nos estudos, acerca das barreiras para seguimento do plano prescrito no controle glicêmico do diabetes sugerem alimentação saudável como dieta cara; alimentos com pouca acessibilidade; baixa assistência familiar; dificuldades em entender a dieta ; falta de informação adequada; dificuldades de se alimentar fora de casa e principalmente recusa em fazer alterações nos hábitos alimentares (MOURA PC, et al., 2018; LANDA-ANELL MV, et al., 2020). Além disso, relatam a restrição alimentar como principal fator limitante (RODRIGUEZ MTG, et al., 2014). 
Os comportamentos como: "beliscar" durante as refeições, comer legumes e verduras), comer em menor quantidade, alimentar-se do que não gosta, e mudança nos horários das refeições são os mais predominantes nos estudos (MOURA PC, et al., 2018; RODRIGUEZ MTG, et al., 2014; LILLO CM e RODRÍGUEZ PP, 2018; SANTOS TBM e FREITAS BJSA, 2018).

A Sociedade Brasileira de Diabetes (2019), estabelece como diretriz que a prescrição de macronutrientes do paciente diabético deve seguir a seguinte divisão:45 a 60\% em carboidratos, proteínas de 15 a $20 \%$ e lipídeos até $30 \%$ do VET total. Todavia, o plano alimentar deve compor uma alimentação saudável, rica em vitaminas, minerais e água. Fibras em quantidades adequadas, para promover o bom funcionamento do intestino e colaborar para a diminuição do índice glicêmico dos alimentos. O plano alimentar do paciente deve considerar suas ingestões habituais, a contagem dos carboidratos diariamente e ser associado a terapia com insulina (SBD, 2019).

Para a adesão é necessário que o paciente esteja comprometido na alimentação adequada, no controle diário da sua glicemia, entender sobre os alimentos e sua carga glicêmica, e da continuidade ao tratamento da diabetes, em vista que se trata de uma doença crônica. Porém, seguir o plano alimentar modificando a sua ingestão usual, usar a medicação, e fazer exercícios ainda é um problema presente na prática clínica (FARIA HTG, et al., 2014)

Diversas são as variáveis capazes de influenciar o paciente a não aderir o tratamento. Fatores pessoais, como por exemplo: conhecimento e percepção acerca da doença, crenças, expectativas futuras sobre a saúde, motivação e capacidade para se comprometer com o tratamento. Por vezes, esses fatores envolvem múltiplas dimensões, como condições sociais, por exemplo convivência com pessoas próximas que sofrem da mesma doença, em que uma influência com apoio e incentivo a outra e proporciona impulso no autocuidado (ASSUNÇÃO TS e URSINE PGS, 2008).

Também deve-se levar em consideração variáveis a respeito da doença: o conhecimento sobre os sinais e sintomas, suas consequências, seus tratamentos efetivos, a consolidação da adesão aos protocolos estabelecidos pelos profissionais de saúde, e principalmente a percepção do risco, em vista que o diabetes inicialmente é assintomático, o que leva aos acometidos a verem como uma patologia de baixa gravidade (ASSUNÇÃO TS e URSINE PGS, 2008).

Outro fator importante é a situação econômica e social em que este paciente está inserido. Renda, desemprego, ausência de rede de apoio familiar, analfabetismo, não acesso a distribuição de medicação gratuita, e alto custo da medicação são variáveis persistentes na baixa da adesão do tratamento do diabetes. (ESTRELA KCA, et al., 2017)

Um plano alimentar bem elaborado, deve levar em consideração os hábitos alimentares individualmente, fatores culturais e emotivos, condição socioeconômica, e acesso ao alimento. Contudo, alterações habituais não são tarefas fáceis para o paciente, envolvem mais do que fatores nutricionais, mas também valores atrelados a cultura, tradições e questões sociais (SANTOS AFL e ARAUJO JWG, 2011).

A dietoterapia do paciente portador de diabetes mellitus tipo 2 torna-se complexa por envolver a presença de sentimentos que influenciam diretamente no tratamento da doença. SALES-PERES SHC, et al. (2016) demostrou que, raiva, ansiedade desânimo, privação de prazer, não aceitação da doença, e preocupação são emoções pertinentes na vida do paciente diabético. O impulso de fazer o que deseja culminado com o sentimento de que deveria fazer o que foi orientado gera um forte sentimento de ansiedade, e sensação de prisão dentro do tratamento (MOURA PC, et al., 2018). Na tese de Arevalos GNB (2015), foi verificado que $51 \%$ dos entrevistados se sentiam tristes, e limitados dos seus prazeres habituais promovidos pela alimentação realizada ao longo da vida.

Uma das estratégicas para atenuar a sensação de desânimo no tratamento do paciente diabético é evidenciado por Santos TBM e Freitas BJSA (2018), que revela adoção de intervenção motivadora não somente para os pacientes, mas para família e equipe multiprofissional, sendo requisito fundamental para 0 estilo de vida. A motivação está diretamente relacionada com maior adesão ao tratamento, combinado com disposição para mudança no estilo de vida, reconhecimento da doença e apoio familiar. 
A rede de apoio familiar também pode ser um fator interferente para o tratamento efetivo do diabetes. A rede de apoio é considerável para a conscientização acerca das mudanças necessárias no tratamento. No estudo de Lillo CM e Rodríguez PP (2018), 68,8\% dos entrevistados afirmaram não serem os responsáveis pela sua alimentação, ficando a cargo dos parceiros, filhos e cuidadores. Elucidando o papel da orientação nutricional não somente restrita ao paciente, mas também para a sua família.

Depois do diagnóstico de DM2, o esperado é que toda a família esteja envolvida e comprometida no tratamento da doença, estabelecendo parceiras no foco com a alimentação e na administração dos medicamentos. Muitos autores sugerem a relação familiar como forte aliado a adesão do tratamento. Quando há organização e apoio dentro do lar, há um melhor controle metabólico (INGERSKI LM, et al., 2010).

Nos estudos realizados, foi possível observar maior adesão ao tratamento farmacológico do que ao seguimento das orientações nutricionais. Groff DP, et al. (2011) em sua análise constatou que $91 \%$ dos entrevistados responderam que realizam o tratamento farmacológico, enquanto somente $50 \%$ informaram seguir as orientações nutricionais prescritas. Evidenciando que de fato, há a necessidade de ampliar as ações de educação em nutrição e implementar estratégias de promoção a saúde para o enfrentamento dos desafios impostos pela falta de adesão.

\section{CONSIDERAÇÕES FINAIS}

A adesão ao tratamento dietoterápico de pacientes diabéticos é baixa $(<50 \%)$. Pelo tratamento complexo da doença se torna um grande desafio para os nutricionistas, no que se refere não somente as mudanças nas práticas alimentares, mas também o contexto em que este individuo está inserido, logo o paciente deve ser analisado sob uma ótica mais integral, considerando aspectos emocionais, culturais, financeiros e sociais. Nesse sentido programas de educação nutricional com abordagens menos tecnicistas, escuta qualificada, valorização da cultura e do cotidiana são mais do que necessárias para o planejamento alimentar do paciente diabético.

\section{REFERÊNCIAS}

1. ANDRADE RC, et al. Prevalence of diabetes mellitus in the Japanese-Brazilian community of Mombuca, Guatapara, SP. Arq Bras Endocrinol Metabol. 2011, 55(2): 127-133.

2. ARAUJO LMB, et al. Tratamento do Diabetes Mellitus do Tipo 2: Novas Opções. Arq Bras Endrocrinol Metab vol 44 , dez. 2000.

3. ARAUJO CC, et al. Internações por diabetes mellitus no estado do Pará: distribuição espacial e fatores associados ao óbito. Revista Nusing. 2019, 22(257): 3223-3233.

4. AREVALOS GNB. Estilo de vida en pacientes con diabetes mellitus tipo 2 que asisten al programa regional de diabetes del hospital regional de encarnación utilizando laencuesta imevid. Universidad nacional de itapúa facultad de medicina. Dissertação (Mestrado em Nutricion Clinica) - Universidad Nacional de Itapúa Facultad de Medicina, Encarnaciónparaguay, 2015.

5. ARRUDA GO, et al. Internações por diabetes mellitus e a Estratégia Saúde da Família, Paraná, Brasil, 2000 a 2012. Ciência e Saúde Coletiva. 2018; 23(2): 543-552.

6. ASSUNÇÃO TS, URSINE PGS. Estudo de fatores associados á adesão ao tratamento não farmacológico em portadores de diabetes mellitus assistidos pelo Programa saúde da Família, Ventosa, Belo Horizonte. Ciência e Saúde Coletiva, 2008, 13(2): 2189-2197.

7. BARBOSA MAG, et al. Alimentação e diabetes mellitus: percepção e consumo alimentar de idosos no interior de pernambuco. Rev Bras Promoç Saúde, Fortaleza, 2015, 28(3): 370-378.

8. BRASIL. Secretaria de Governo do Estado do Pará. Boletim Epidemiológico. 2019.

9. BRASIL. Ministério da Saúde. Secretária de Vigilância em Saúde. Boletim Epidemiológico. Volume 51, abril 2020.

10. COSTA JA, et al. Promoção da saúde e diabetes: discutindo a adesão e a motivação de indivíduos diabéticos participantes de programas de saúde. Ciência \& Saúde Coletiva, 16(3):2001-2009, 2011.

11. DIAS, et al. O cuidado em enfermagem direcionado para a pessoa idosa: revisão integrativa. Revista de Enfermagem UFPE Online, Recife, 2014, 5(8): 1337-1346.

12. DIAS JCR, CAMPOS JADB. Epidemiological aspects of obesity and its relation to Diabetes mellitus. Nutrire: rev. Soc. Bras. Alim. Nutr. J. Brazilian Soc. Food Nutr., São Paulo, SP, 2008, 33(1): 103-115. 
13. DURCO ES. Protocolo de tratamento do paciente adulto jovem com diabetes mellitus tipo 2. Belo Horizonte, Minas Gerais, 2009.

14. ESTRELA KCA, et al. Adesão às orientações nutricionais: uma revisão de literatura. Demetra: alimentação, nutrição \& saúde. 2017.

15. FARIA HTG, et al. Adesão ao tratamento em diabetes mellitus em unidades da Estratégia Saúde da Família. Ver Esc Enferm USP 2014; 48(2): 257-63.

16. GOMES-VILLAS BOAS LC, et al. Relação entre apoio social, adesão aos tratamentos e controle metabólico de pessoas com diabetes mellitus. Revista Latino Americana de Enfermagem, jan./fev. 2012; 20(1).

17. GROFF DP, et al. Adesão ao tratamento dos pacientes diabéticos tipo II usuários da estratégia saúde da família situada no bairro Metropol de Criciúma, SC. Arquivos Catarinenses de Medicina. 2011; 40(3).

18. INGERSKI LM, et al. Blood glucose monitoring and glycemic control in adolescence: contribution of diabetes-specific responsibility and family conflict. J Adolesc Health 2010; 47(2): 191-7.

19. INTERNACIONAL DIABETES FEDERATION. Atlas IDF 2017: Diabetes no brasil. 2017. Disponível em: https://www.diabetes.org.br/profissionais/images/2018/poster-atlas-idf-2017.pdf. Acesso em: 14 nov. 2020.

20. KLAUTAU FC, et al. Situação de diabetes em Belém, Ananindeua, Pará e Brasil. Anais do CBMFC, 2013.

21. LANDA-ANELL MV, et al. Barriers to adherence to a nutritional plan and strategies to overcome them in patients with type 2 diabetes mellitus; results after two years of follow-up. Endocrinol Diabetes Nutr. 2020; 67(1): 4-12.

22. LOTTENBERG AMP. Características da Dieta nas Diferentes Fases da Evolução do Diabetes Melito Tipo 1. São Paulo, Arq Bras Endrocrinol Metab. 2008; 52(2).

23. LILLO CM, RODRÍGUEZ PP. Valoracion de la adherencia del paciente diabético al consejo nutricional y evaluación de mejoras tras su reeducación: una investigación-acción en la costa mediterrânea. Rev Chil Nutr 2018; 45(3): 205215.

24. MOURA PC, et al. Educação nutricional no tratamento do diabetes na atenção primária à saúde: vencendo barreiras. rev. aps. $2018 \mathrm{abr} / \mathrm{jun} ; 21(2): 226-234$.

25. OLIVEIRA LMSM, et al. Adesão ao tratamento dietético e evolução nutricional e clínica de pacientes com diabetes mellitus tipo 2. HU Revista, Juiz de Fora, 2016, 42(4): 277-282.

26. OPAS. Dia Mundial da Saúde 2016: Combater o diabetes. Disponível em: https://www.paho.org/bireme/index.php?option=com_content\&view=article\&id=326:dia-mundial-da-saude-2016combater-o-diabetes\&ltemid=183\&lang=pt. Acesso em: 25 set. 2020.

27. POPKIN BM. The nutrition transition and obesity in the developing world. Journal of Nutrition, 2001,131: 871-873

28. REBELO AB, VAZ EM. Associação de Diabetes Mellitus com transtornos alimentares. Nutrire. 2014 Aug; $39(2)$ : $252-$ 264.

29. RODRIGUEZ MTG, et al. Adesão ao aconselhamento nutricional para o diabetes mellitus em serviço de atenção primária á saúde. Revista Mineira de Enfermagem. 2014 jul/set; 18(3): 685-690.

30. SALES-PERES SHC et al. Estilo de vida em pacientes portadores de diabetes mellitus tipo 1: uma revisão sistemática. Ciênc. Saúde coletiva [online]. 2016, 21(4): 1197-1206.

31. SANTOS AFL, ARAUJO JWG. Pratica alimentar e diabetes: desafios para a vigilância em saúde. Epidemiol. Serv. Saude, Brasilia, 2011, 20(2): 263.

32. SANTOS GFP, et al. A adesão ao autocuidado influencia parâmetros bioquímicos e antropométricos de pacientes diabéticos tipo 2 atendidos no programa hiperdia do município de Juiz de Fora, Minas Gerais, Brasil.Nutr. clín. diet. hosp. 2014; 34(3): 10-19.

33. SANTOS TBM, FREITAS BJSA. Adesão ao tratamento dietético em portadores de diabetes mellitus assistidos pela estratégia saúde da família. BRASPEN J. 2018; 33(1): 76-85.

34. SARTORELLI DS, CARDOSO MA. Associação entre carboidratos da dieta habitual e diabetes mellitus tipo 2 : evidências epidemiológicas. Arq Bras Endocrinol Metabol. 2006; 50(3): 415-26.

35. SILVA A. Diretrizes da sociedade brasileira de diabetes 2009-2020/Sociedade brasileira de diabetes. Itapevi: São Paulo; 2019.

36. VERMELHO LL, et al. Transição epidemiológica e indicadores de saúde atuais. Cad. Saúde Coletiva. 2011, 9(2): 111126.

37. VIGITEL BRASIL. Ministério da Saúde. 2017. Disponível em: http://bvsms.saude.gov.br/bvs/publicacoes/vigitel_brasil 2017_vigilancia_fatores_riscos.pdf. Acesso em: 27 set. 20.

38. ZANETTI ML, et al. Adesão às recomendações nutricionais e variáveis sociodemográficas em pacientes com diabetes mellitus. Rev Esc Enferm USP . 2015; 49(4): 619-625.

39. WHO. WORLD HEALTH ORGANIZATION. Adherence to long-term therapies: evidence for action. Geneva: WHO; 2003.

40. WHO. WORLD HEALTH ORGANIZATION. Noncommunicable diseases country profiles 2018. Geneva, 2018. 